\title{
Emission Tax or Standard? The Role of Productivity Dispersion*
}

\author{
Zhe Li \\ Shanghai University of \\ Finance and Economics \\ (li.zhe@mail.shufe.edu.cn)
}

\author{
Shouyong Shi \\ Department of Economics \\ University of Toronto \\ (shouyong@chass.utoronto.ca)
}

This version: May 2012

\begin{abstract}
We analyze the importance of productivity dispersion for evaluating a tax versus a regulatory standard on emission. The model has a continuum of plants each producing a dirty good with emission as the by-product. The plants are heterogeneous in productivity, and the dirty-goods sector is monopolistically competitive. We show that if no technology is available for emission abatement, then the tax generates higher welfare than the standard for any given target on aggregate emission. With an abatement technology, the standard can yield higher welfare than the tax if the plants have sufficiently large market power and sufficiently similar productivity.
\end{abstract}

Keywords: emission tax, standard, productivity dispersion, abatement.

*The research reported here was not the result of a for-pay consulting relationship. Neither we nor our employers have a financial interest in the topic of the paper which might constitute a conflict of interest. We have received useful comments from the participants of the Society for Economic Dynamics meeting (2010), the World Congress of the Econometric Society (2010), the Midwest Macro Conference (2010) and Shanghai Macro Workshop (2010). Li would like to acknowledge financial support by the Shanghai Pujiang Program, the Shanghai Leading Academic Discipline Project (number B801), and the 211 Project. Shi would like to acknowledge financial support from the Canada Research Chair, the Bank of Canada Fellowship, and the Social Sciences and Humanities Research Council of Canada. The opinion expressed here is the authors' own and does not reflect the view of the Bank of Canada. 


\section{Introduction}

The economics of climate change has attracted increasing attention, especially after the publication of the Stern (2006) Review. There is wide disagreement on how stringent the emission target should be in order for the world to avoid a large loss that can be caused by climate change. In this paper, we address a different question: Given a target on aggregate emission, is it more desirable to achieve it by imposing a tax or a nontradable standard on emission? With a model of monopolistic competition, we demonstrate that the answer depends critically on the extent of productivity dispersion in the economy.

Regulatory standards and market-based instruments are two common forms of environmental policy. A regulatory standard specifies with a certain degree of precision the actions that a firm or individual must take to achieve environmental objectives. Such a standard cannot be met by trading in the market. In contrast, instruments like emission taxes and tradable emission quotas or permits work through the market. Traditionally, regulatory standards were predominant. Starting from the 1970s, however, opinions have been shifting to favor market-based instruments. For example, the Stern Review (part IV, p310) states that "a common price signal is needed across countries and sectors to ensure that emission reductions are delivered in the most cost-effective way..... [Both] taxes and tradable quotas have the potential to deliver emission reductions efficiently." ${ }^{1}$

We contribute to this policy debate by demonstrating the importance of productivity dispersion among firms. Although the literature on environmental policy has discussed firm heterogeneity, it has not focused on rich dispersion in productivity across firms. ${ }^{2}$ This lack of focus is problematic. First, how environmental policy shifts resources between firms or regions that differ in productivity should be important for policy evaluation. Second, rich dispersion in productivity is important in accounting for trade flows and the effects

\footnotetext{
${ }^{1}$ Freeman and Kolstad (2007) document the past twenty years of experience in using market-based instruments, in comparison with command-and-control policies such as regulatory standards.

${ }^{2}$ On firm heterogeneity, some papers have incorporated cost heterogeneity, but they have restricted heterogeneity to only a few values in the context of duopoly or oligopoly (e.g., Simpson, 1995, Shaffer, 1995, Carraro and Soubeyran, 1996, and Fischer, 2011). In contrast, our model has a continuum of monopolistically competitive firms and a continuous distribution of productivity levels across the firms.
} 
of trade policies (e.g. Eaton and Kortum, 2002, and Melitz, 2003). One should expect productivity dispersion to be even more important for environmental policies. Emission creates externalities globally, not just to the region where emission is produced. With this world-wide view, it is easy to recognize that different countries have vastly different levels of productivity. Even if an environmental policy is applied to all countries with no leakage, its effect on emission and welfare should depend critically on how firms with different productivity respond to the policy in a general equilibrium.

To uncover the importance of productivity dispersion, we introduce emission and environmental policies into a model of monopolistic competition. There are two types of consumption goods: a clean good and a dirty-goods composite. The clean good generates no emission and is produced by a perfectly competitive sector. The dirty-goods composite aggregates a continuum of imperfectly substitutable varieties. The dirty-goods sector is monopolistically competitive, with each plant producing a distinct variety. A plant's productivity is a draw from a continuous distribution over a wide range of values. The production of a dirty good generates emission as a by-product, and aggregate emission reduces the households' utility. A plant's emission increases with its input and, hence, with its output. However, a more productive plant has a lower emission intensity, i.e., a lower emission-output ratio. A plant may undertake emission abatement.

We evaluate two environmental policies. One is a standard that requires a plant's emission-intensity not to exceed a given level, and the other is a tax imposed on each unit of emission. ${ }^{3,4}$ Given any target on the aggregate level of emission, we compare the equilibria under the two policies. To isolate the role of productivity dispersion, we first examine the economy where no abatement technology is available and then introduce an abatement technology. In each economy, we determine equilibrium quantities and prices

\footnotetext{
${ }^{3}$ The regulatory standard examined here is a performance standard instead of a technology standard (see IPCC, 2007). A technology standard mandates specific pollution abatement technologies or production methods, such as specific $\mathrm{CO}_{2}$ capture and storage methods on a power plant. A performance standard mandates specific environmental outcomes per unit of product (or input) such as a certain number of grams of $\mathrm{CO}_{2}$ per kilowatt-hour of electricity generated.

${ }^{4}$ We do not examine tradable emission permits or quotas because they are equivalent to the emission tax in our model. In fact, the price of such tradable permits is equal to the tax rate. Monnet and Temzelides (2011) examine more elaborate emission trading mechanisms.
} 
of goods produced in the two sectors. We rank the two policies with the representative household's utility as the welfare function.

A critical difference between the two policies is their effect on the average productivity in the dirty-goods sector. The tax does not change the average productivity, because it increases all plants' marginal costs by the same proportion and reduces each plant's input and output by the same proportion. In contrast, the standard constrains low-productivity plants more severely because low-productivity plants have a higher emission intensity. This uneven constraint across the plants shifts resources from low-productivity to highproductivity plants. A natural conjecture is that this shift increases the average productivity in the sector, which may make the standard a better policy than the tax. Surprisingly, the opposite is true: The shift in resources reduces the average productivity.

This result arises from the feature that the variety produced by a plant with higher productivity has a lower marginal contribution to the dirty-goods composite because this marginal contribution is diminishing. The average productivity in the economy is a weighted sum of the plants' productivity, where the weights for a plant are the variety's marginal contribution to the composite. ${ }^{5}$ This "value-weighted" marginal productivity is equalized across all operating plants in the equilibrium as a result of perfect mobility of resources across the plants. When the standard shifts resources from low-productivity plants to high-productivity plants, it increases output of the plants whose output is already high and whose marginal contribution to the composite is low. Thus, the average productivity is lower under the standard than under the tax. This result holds regardless of whether or not the abatement technology is available. Without the abatement technology, the tax is unambiguously better than the standard. The higher average productivity under the tax not only allows the average price to be lower and output of the dirty-goods composite to be higher, but also induces more varieties of the dirty goods to be produced under the tax.

With the abatement technology, the average productivity is still higher under the tax than under the standard. However, if productivity dispersion is small, the standard may

\footnotetext{
${ }^{5}$ The marginal contribution is used as the weights for a variety in calculating the average productivity because there is a continuum of varieties, each of which contributes only marginally to the composite.
} 
induce a smaller increase in the marginal cost of production than the tax, as we will explain in section 4.3. If this cost advantage is sufficiently large to outweigh the disadvantage in the average productivity, then the standard generates higher output of the dirty goods than the tax, at the expense of the clean good. The quantity of the dirty goods is significantly higher under the standard than under the tax only if a relatively small saving in the marginal cost translates into a large increase in output. This is the case when the plants have sufficiently high market power. Thus, the relative advantage of the two policies depends on productivity dispersion and market power. For the standard to generate higher welfare than the tax, the plants need to have both sufficiently high market power and sufficiently similar productivity. If either market power is small or productivity dispersion is large, then the tax dominates the standard. Moreover, when productivity dispersion exceeds a certain level, the tax is superior to the standard for all values of market power.

Our analysis implies that the wider the sector or region that an emission target is intended to cover, the more likely that the tax achieves higher welfare than the standard. Especially, when a target covers many countries or the entire world, the vast dispersion in productivity can render the tax a far better policy than the standard. Thus, after all, the shift of opinions in the last four decades or so in favor of market-based instruments does have a justification on the ground of productivity dispersion.

Productivity dispersion is modeled here similarly to the Eaton-Kortum-Melitz trade model. As such, the modeling builds a link between environmental and international economics. Despite this link, we should caution that environmental issues are different from trade issues such as tariffs and quotas (e.g., Young and Anderson, 1980). First, emission generates a negative externality to the society which has no apparent counterpart in the trade literature. Second, emission is a by-product of production. An emission policy is imposed on this by-product rather than on the regular goods as are tariffs and trade quotas. Third, the producers can reduce emission by abatement without affecting output of the regular goods, and different environmental policies affect the abatement choice differently.

Our paper is related to the literature originated from the seminal paper of Weitzman 
(1974) that addresses the question whether it is more desirable to control the price or the quantity of emission. ${ }^{6}$ The main issue in our paper is not price-versus-quantity, since we fix the quantity of aggregate emission at any arbitrary target. Moreover, the price-versusquantity literature evaluates one group of market (price) instruments against another group of market instruments (e.g., tradable quotas). In contrast, we evaluate a market instrument (the tax) against a nontradable instrument (the standard). Finally, while uncertainty is the main character in this literature that determines whether a price or a quantity control is superior, we abstract from aggregate uncertainty and focus, instead, on productivity dispersion among the plants and its interaction with abatement.

Since the plants have monopoly power on a variety, our model is related to the literature, originated from Buchanan (1969), that analyzes the importance of the market structure in determining optimal corrective policy in the presence of externalities. The main insight of this literature is that when market power causes output to be inefficiently low, a tax on firms exacerbates the distortion by further reducing output, in which case policies that do not directly target prices (such as a standard) may be better in correcting the inefficiency. ${ }^{7}$ In contrast to this literature, we focus on productivity dispersion rather than market power. Rich dispersion in productivity changes the main results significantly. In particular, when an abatement technology is not available, the tax always generates higher welfare than the standard no matter how high market power is. When an abatement technology is available, market power can yield an advantage to the standard over the tax that is consistent with the above insight, but this advantage exists only if productivity dispersion is small. When productivity dispersion exceeds a critical level, the tax, again, generates higher welfare than the standard regardless of how large market power is.

Let us clarify our focus further. First, we assume that a government can only choose

\footnotetext{
${ }^{6}$ We do not survey this literature here. For some examples, see Laffont (1977) for incorporating subjective uncertainty, Yohe (1978) for examining additional sources of uncertainty and informational difficulty within a regulated heirarchy, and Kelly (2005) for a general-equilibrium framework. Other examples are Pizer (2002), Hepburn (2006) and Mandell (2008).

${ }^{7}$ See Requate (2005) for a survey. The early examples in this literature are Baumol and Oates (1975) and Barnett (1980). Some later examples are Simpson (1995), Shaffer (1995), Carraro and Soubeyran (1996) and Holland (2009).
} 
either the emission tax or the emission-intensity standard, instead of a cocktail of policies, and the tax revenue is rebated to consumers through lump-sum transfers. ${ }^{8}$ Second, we compare the two policies under any given emission target instead of the optimal target. However, our results hold even if the target is optimally set. If one policy is better than another policy under all arbitrarily given emission targets, it is also better if the target is chosen optimally. Third, we abstract from many other factors such as emission monitoring when producers have private information (see Montero, 2005) and practical difficulties or leakages in implementing a policy (see Stern, 2006). Such abstraction is intended to focus on productivity dispersion whose role is not well understood in the literature in the evaluation of environmental policies.

\section{Model Environment}

Consider a one-period economy that is populated by a unit measure of households. Each household is endowed with one unit of resource that can be supplied in production and receives dividends from a diversified portfolio of the plants. A household's utility function is $u(c, Q, M)$, where $c$ is consumption of a clean good, $Q$ consumption of a composite of the dirty goods, and $M$ the aggregate level of emission per household. Define

$$
R(c, Q, M)=\frac{u_{2}(c, Q, M)}{u_{1}(c, Q, M)},
$$

where the subscripts of $u$ indicate partial derivatives.

Assumption 1 (i) $u_{1}>0, u_{2}>0, u_{11}<0$ and $u_{22}<0$; (ii) $u_{3}<0$; (iii) $R_{3}(c, Q, M) \leq 0$.

Part (i) of the assumption is standard. Part (ii) says that emission generates a negative externality on households. Part (iii) says that emission (weakly) increases a household's desire for the clean good relative to the dirty goods.

\footnotetext{
${ }^{8}$ This assumption eliminates the need for government revenue as a potential difference between the tax and the standard. It also eliminates output-based refunding schemes and the so-called double dividend of a policy that arises from using the revenue of the policy to reduce other distortions in the economy. See Stern (2006, Part IV), Gersbach and Requate (2004) and Fischer (2011) for more discussions.
} 
The clean good is homogeneous and its production does not generate emission. The technology of producing the clean good is $w \ell_{c}$, where $\ell_{c}$ is the input and $w>0$ is a constant. For the sake of simplicity, we lump all types of inputs into one so that the production function is linear in this input. There is perfect competition in the clean-good sector, and so the price of the resource in terms of the clean good is equal to the constant $w$. The clean good serves as the numeraire in this model. ${ }^{9}$

The dirty-goods sector is monopolistically competitive and produces varieties in the continuum, $[0,1]$. Each variety is produced by at most one plant, and the range of varieties produced in the equilibrium is endogenous. All plants in the dirty sector are identical ex ante. Ex post, they are heterogeneous because of their choice of the variety and a random draw of productivity. At the beginning of the period, each plant can choose at most one variety to produce. It is optimal for different plants to choose distinct varieties. After this choice, the plant draws a productivity level $x$ from the distribution (cdf) $G($.$) ,$ with a support $[\underline{x}, \infty)$. Then, a plant chooses whether or not to operate. If a plant with productivity $x$ operates, its output is

$$
q(x)=e^{x} \ell(x),
$$

where $\ell(x)$ is the plant's input. Because the plant is the only producer of the variety, it takes the demand curve for the variety, not the price of the variety, as given. We refer to a plant with productivity $x$ as plant $x$.

Whether a plant chooses to operate or not can depend on the environmental policy in place. Hence, the set of productivity levels in operation, denoted as $X$, may not necessarily be the same as the support of the distribution $G$. The composite of the dirty goods that enters a household's utility function is:

$$
Q=\left[\int_{x \in X}[q(x)]^{\frac{\varepsilon-1}{\varepsilon}} d G(x)\right]^{\frac{\varepsilon}{\varepsilon-1}}, \quad 1<\varepsilon<\infty .
$$

\footnotetext{
${ }^{9}$ The assumption that the clean-good sector is perfectly competitive and generates no emission is made without loss of generality. Including the clean good in the model enables us to capture a policy's generalequilibrium effect of shifting resources between the two sectors. Also, it enables us to model all fixed costs and taxes in terms of the clean good, thereby simplifying the accounting in the model.
} 
The constant $\varepsilon$ is the elasticity of substitution between any two varieties. A plant's "market power", $1 /(\varepsilon-1)$, is finite because $\varepsilon>1$, and positive because $\varepsilon<\infty$. It is important to bear in mind that two plants can draw the same $x$ but they necessarily produce different varieties. The output levels of different plants are not additive in the aggregator (2), regardless of whether they draw the same or different productivity. ${ }^{10}$

Production of a dirty good generates emission as a by-product. In the baseline model, the amount of emission of plant $x$ obeys the following process:

$$
m(x)=b \ell(x), \quad b>0 .
$$

In an extended model, we will introduce emission abatement. Let us express the abatement level, $a$, in terms of the input so that the abatement cost is wa. A plant $x$ that chooses abatement $a(x)$ has the following level of emission:

$$
m(x)=b \ell(x)\left(1+\frac{a(x)}{\ell(x)}\right)^{-\frac{1}{\gamma}}, \quad \gamma>0 .
$$

The effectiveness of the abatement technology can be measured by $1 / \gamma$. In the limit $\gamma \rightarrow 0$, even a tiny amount of abatement can eliminate the plant's emission; in the opposite limit $\gamma \rightarrow \infty$, abatement does not reduce emission. We refer to the ratio $m(x) / q(x)$ as the emission intensity, and to the ratio $a(x) / \ell(x)$ as the abatement intensity.

This emission process captures two general and realistic features. First, given the same abatement intensity, a high- $x$ plant has a lower emission intensity than a low- $x$ plant, although the level of emission increases in $x$ as output does. The use of this feature will become apparent below. Second, the emission intensity is decreasing and convex in the abatement intensity. It is intuitive that the emission intensity is a function of the intensity, rather than the level, of abatement. This function should be decreasing in order for the abatement intensity to have a positive marginal contribution to the reduction in the emission intensity, and convex in order for this marginal contribution to be diminishing. Although our main results can continue to hold with more general emission processes that have these features, the simple form above maintains tractability.

\footnotetext{
${ }^{10}$ The quantity $q(x)$ is not total output of all plants that draw the same $x$, but rather the output of each of these plants. The same clarification applies to all plant-specific notation, such as $\ell(x)$.
} 
We evaluate two environmental policies: (i) an emission tax $\tau$ that requires a plant to pay $\tau$ (in terms of the clean good) for every unit of emission, and (ii) an emission standard $s$ that requires a plant's emission intensity not to exceed $s$. The revenue from the tax is rebated to the households through lump-sum transfers. Note that the emission standard is on a plant's emission intensity rather than the level. This specification makes sense when the plants are heterogeneous in productivity. A standard on the emission level, instead, would constrain a high-productivity plant more than a low-productivity plant. Similarly, the presence of heterogeneous productivity is the reason why we assume that a plant's emission intensity is a decreasing function of $x$. If a plant's emission intensity is an increasing function of productivity, instead, then a standard puts an upper bound on productivity below which a plant can operate. Since a standard in this case prevents more productive plants from operating, it does not seem to be a good policy.

Another commonly debated policy is one that requires a plant to obtain an emission permit for each unit of emission. We do not examine this policy separately here because it is equivalent to the tax in our model, as stated below:

Remark 2 When an emission permit is tradable in a competitive market, it is equivalent to an emission tax, with the price of the permit being equal to the tax rate.

Moreover, a standard can be interpreted as a nontradable permit that is granted free to the plants whose emission intensity does not exceed the constant $s$. Thus, an emission tax differs from an emission standard in two ways. One is that the tax is a market instrument but the standard is nontradable. The other is that the tax directly affects a plant's marginal cost of production, but the standard affects the marginal cost only indirectly through the abatement choice and/or equilibrium effects. ${ }^{11}$

Note that uncertainty does not play an important role in this model, in contrast to the literature on price versus quantity. Although a plant's productivity is random, it is realized

\footnotetext{
${ }^{11}$ Another policy is to issue a production permit (license) that a plant needs to produce dirty goods and that can be sold in the market at a competitive price. In contrast to an emission tax or standard, such production permits do not directly restrict a plant's emission, because a plant that obtains a production permit can produce as much as it wants. For this reason, a production permit cannot affect a plant's choice of the abatement technology.
} 
before the plant chooses input, output and abatement. Moreover, the continuum of plants ensures that there is no aggregate uncertainty. Specifically, the supply curve of the dirtygoods composite, total input, total abatement, and total emission are all deterministic.

\section{Equilibrium and Policies without Abatement}

It is important to isolate the role of productivity dispersion in policy evaluation. For this purpose, we first examine the economy where an abatement technology does not exist so that emission follows (3). Section 4 will incorporate an abatement technology. In the current section, we unify the notation for the equilibrium when both policies are present.

\subsection{A household's decisions}

A household chooses consumption of the clean good, $c$, and consumption of the dirty goods, $(q(x))_{x \in X}$, where $q(x)$ is the demand for the variety of each plant that draws productivity $x$. Let $p(x)$ be the price of the variety produced by plant $x$. The household's optimal choices maximize $u(c, Q, M)$ subject to (2) and the following budget constraint:

$$
c+\int_{x \in X} p(x) q(x) d G(x) \leq w+\int_{x \in X} \pi(x) d G(x)+T .
$$

Here, $\pi(x)$ is the dividend from plant $x$ and $T$ the lump-sum transfer from the government. This maximization problem yields the following optimality conditions:

$$
\begin{gathered}
p(x)=P\left(\frac{q(x)}{Q}\right)^{-1 / \varepsilon}, \\
\frac{u_{2}(c, Q, M)}{u_{1}(c, Q, M)}=P,
\end{gathered}
$$

where $P$ is the following price of the composite $Q$ :

$$
P=\left[\int[p(x)]^{1-\varepsilon} d G(x)\right]^{\frac{1}{1-\varepsilon}} .
$$

\subsection{Plants' decisions}

Consider a plant $x$ in the dirty-goods sector that chooses to produce. The plant's profit is:

$$
\pi(x)=p(x) q(x)-w \ell(x)-\tau m(x) .
$$


The plant faces the demand curve for its product, given by (5). It chooses the input, $\ell(x)$, to maximize profit, taking as given the demand for the composite, $Q$, and the price index, $P$. Substituting $p(x)$ from (5), $q(x)$ from (1), and $m(x)$ from (3), we get:

$$
\pi(x)=P Q^{\frac{1}{\varepsilon}}\left[e^{x} \ell(x)\right]^{\frac{\varepsilon-1}{\varepsilon}}-(w+\tau b) \ell(x) .
$$

It is easy to verify that the plant's optimal input is

$$
\ell(x)=Q e^{(\varepsilon-1) x}\left(\frac{(\varepsilon-1) P}{\varepsilon(w+\tau b)}\right)^{\varepsilon} .
$$

The plant's output is $q(x)=e^{x} \ell(x)$ and its emission is $m(x)=b \ell(x)$. The price of the plant's product, given by (5), can be written explicitly as

$$
p(x)=\frac{\varepsilon}{\varepsilon-1}(w+\tau b) e^{-x} .
$$

The term $(w+\tau b) e^{-x}$ is the plant's effective marginal cost, which is the marginal cost adjusted for the plant's productivity and the emission tax. Thus, the price is a constant markup $\frac{1}{\varepsilon-1}$ over the effective marginal cost. Moreover, the plant's maximized profit is:

$$
\pi(x)=\frac{P^{\varepsilon} Q}{\varepsilon} e^{(\varepsilon-1) x}\left(\frac{\varepsilon-1}{\varepsilon(w+\tau b)}\right)^{\varepsilon-1} .
$$

A plant's decision on whether or not to operate follows a cutoff rule; that is, there exists $x_{0} \in[\underline{x}, \infty)$ such that a plant $x$ operates if and only if $x \geq x_{0}$. The cutoff $x_{0}$ depends on the policy. Under the emission tax, since $\pi(x)>0$ for all $x$, then $x_{0}=\underline{x}$; that is, all plants choose to operate. Under the emission standard, a plant $x$ can operate if and only if $s \geq m(x) / q(x)=b e^{-x}$; that is, $x_{0}=\ln (b / s)$. We summarize these two cases:

$$
x_{0}= \begin{cases}\ln (b / s), & \text { with the emission standard } \\ \underline{x}, & \text { with the tax. }\end{cases}
$$

The set of productivity levels observed in the economy is $X=\left[x_{0}, \infty\right)$.

\subsection{Aggregation and equilibrium}

Let us denote total input in the dirty-goods sector as $L=\int_{x_{0}}^{\infty} \ell(x) d G(x)$ and the average productivity in the dirty-goods sector as $\phi=Q / L$. Substituting $\ell(x)$ from (8) to compute $L$ 
and $q(x)$ from (1) to compute $Q$, we can express the average productivity in the dirty-goods sector as $\phi=\phi\left(x_{0}\right)$ where

$$
\phi\left(x_{0}\right)=\left[\int_{x_{0}}^{\infty} e^{(\varepsilon-1) x} d G(x)\right]^{\frac{1}{\varepsilon-1}} .
$$

Substituting (9) into (7) reveals that the price index of the dirty-goods composite is:

$$
P(\tau, \phi) \equiv \frac{\varepsilon}{\varepsilon-1}\left(\frac{w+\tau b}{\phi}\right) .
$$

If the operating plants' effective marginal costs, $(w+\tau b) e^{-x}$, are aggregated in the same way as the varieties' prices are aggregated for the price index, then the average effective marginal cost is $(w+\tau b) / \phi$. According to (13), the price level of the dirty-goods composite is a constant markup over the average effective marginal cost.

Let us refer to $(\tau, s, \bar{M}, T)$ as the policies, where $\bar{M}$ is the target level of aggregate emission. An equilibrium under the policies $(\tau, s, \bar{M}, T)$ consists of the set $X=\left[x_{0}, \infty\right)$, the functions $(\ell(x), q(x), p(x))_{x \in X}$, and the aggregate levels $(c, Q, P, L, M)$ that satisfy the following requirements: (i) Given the functions $(p(x))_{x \in X}$, a household's demand for the clean good, $c$, and the demand for each dirty good, $q(x)$, satisfy (5) and (6); (ii) Given $(P, Q)$ and the demand function (5), a plant operates if and only if $x \geq x_{0}$, where $x_{0}$ satisfies (11), and if a plant operates, its choices of input and output satisfy (8) and $q(x)=e^{x} \ell(x)$; (iii) The levels of $(P, L, M)$ satisfy (13), $L=Q / \phi$ and $M=b L$, where $\phi$ is given by (12); (iv) The resource market clears, i.e., $\ell_{c}=1-L$; (v) The market of the clean good clears, i.e., $c=w \ell_{c}$, and the markets of the dirty goods clear; (vi) The policy $\tau$ or $s$ ensures aggregate emission not to exceed the target $\bar{M}$, i.e., $M \leq \bar{M}$, while the transfer $T$ satisfies $T=\tau M$ under the tax and $T=0$ under the standard.

We examine the relevant case where the emission target $\bar{M}$ is binding, i.e., where the economy would produce $M>\bar{M}$ if there were no policy. In this case, $M=\bar{M}$ in the equilibrium. An equilibrium can be determined as follows. Part (iii) above gives $L=\bar{M} / b$, $Q=\phi \bar{M} / b$ and $P=P(\tau, \phi)$, while parts (iv) and (v) give $c=w\left(1-\frac{\bar{M}}{b}\right)$. With these results, (6) required by part (i) becomes

$$
R\left(w-w \frac{\bar{M}}{b}, \phi \frac{\bar{M}}{b}, \bar{M}\right)=P(\tau, \phi),
$$


where $\phi=\phi\left(x_{0}\right)$. Equation (14) determines the tax or standard that is needed to implement the emission target $\bar{M}$. Note that the emission standard enters the equation through $\phi\left(x_{0}\right)$, because $x_{0}$ is a function of $s$. For any target $\bar{M}$, let $\tau(\bar{M})$ denote the tax, and $s(\bar{M})$ the standard, that achieves the target.

Lemma 3 Suppose that an abatement technology does not exist. Assume that $[Q R(c, Q, M)]$ is strictly increasing in $Q$ for any given $(c, M)$ and that $\lim _{Q \rightarrow 0}(Q R)=0$. The target $\bar{M}$ is binding if and only if $\bar{M}<M_{\max }$, where $M_{\max }$ is defined by

$$
R\left(w-w \frac{M_{\max }}{b}, \phi(\underline{x}) \frac{M_{\max }}{b}, M_{\max }\right)=P(0, \phi(\underline{x})) .
$$

There is a unique equilibrium under each policy. Moreover, $\tau^{\prime}(\bar{M})<0$ and $s^{\prime}(\bar{M})>0$.

The proof of this lemma appears in Appendix A. In addition to existence and uniqueness of an equilibrium, the lemma states the intuitive feature that the tax can be lower and the standard can be less stringent when the emission target is higher.

\subsection{Comparing the two policies}

Let us add the subscript $\tau$ to $\left(x_{0}, L, P, Q, \phi, u\right)$ under the tax and the subscript $s$ under the standard. The following proposition compares the equilibrium under the tax with the equilibrium under the standard (see Appendix A for a proof):

Proposition 4 Assume that an abatement technology does not exist. Given any binding emission target $\bar{M}$, the following results hold: (i) $L_{\tau}=L_{s}$ and $c_{\tau}=c_{s}$; (ii) $x_{0 \tau}<x_{0 s}$, $Q_{\tau}>Q_{s}, \phi_{\tau}>\phi_{s}$ and $P_{\tau}<P_{s}$; (iii) $u_{\tau}>u_{s}$.

Result (i) is not surprising. Since a plant's emission is proportional to the plant's input, total emission is proportional to total input in the dirty-goods sector. Given the same emission target, the emission process (3) implies that total input in the dirty-goods sector must be the same under the two policies. As a result, total input in the clean-good sector and, hence, consumption of the clean good must also be the same under the two 
policies. If the plants were homogeneous, this result would imply that the two policies were equivalent, because they induce the same resource allocation between the two sectors.

The two policies are not equivalent when the plants are heterogeneous in productivity, as stated in (ii) and (iii) above. They induce different allocations within the dirty-good sector. Relative to the standard, the tax induces a larger set of varieties of the dirty goods to be produced, higher consumption of the dirty-goods composite, a higher average productivity and a lower price of the dirty-goods composite. We will explain these effects below. Since the tax generates higher consumption of the dirty-goods composite and the same level of consumption of the clean good, the tax dominates the standard in welfare.

The outcome $x_{0 \tau}<x_{0 s}$ in (ii) above means that more varieties of the dirty goods are produced under the tax than under the standard. This outcome arises from the difference in how the two policies affect a plant's marginal cost and emission. The tax increases every plant's effective marginal cost of production, thereby reducing every plant's input, output and emission. However, since each plant charges a price that is a constant markup over the effective marginal cost, every plant's profit is still positive after the tax. In contrast, the standard does not affect a plant's effective marginal cost and emission, provided that the plant can meet the standard. To meet the emission target at the aggregate level, some plants must shut down. These are the plants with low levels of $x$. As a result, fewer varieties are produced under the standard than under the tax.

More importantly, the two policies differ in the effect on the average productivity. As is clear from (12), the tax does not change the average productivity, because the tax reduces total input in the dirty-goods sector and output of the dirty-goods composite in the same proportion. In contrast, the standard shuts down low- $x$ plants, and the input released from low- $x$ plants is shifted to high- $x$ plants. A priori, one would guess that this shift should increase the average productivity. Surprisingly, the opposite is true, as (12) clearly shows that the average productivity falls as $x_{0}$ increases. Thus, the average productivity is lower under the standard than under the tax. 
How can it be the case that moving the input from low- $x$ plants to high- $x$ plants reduces the average productivity? The key to answering this question is to understand that a variety is valued by its marginal contribution to the composite, $Q$. This value-weighted marginal productivity of the input is the same for all $x$. To verify this statement, note that the sum of the input in all plants with the same $x$ is $G^{\prime}(x) \ell(x)$. Using (2), (5), (9), and (13), we can compute the marginal contribution of this input to $Q$ as:

$$
\frac{\partial Q}{G^{\prime}(x) \partial[\ell(x)]}=e^{x}\left(\frac{Q}{q(x)}\right)^{1 / \varepsilon}=\frac{\varepsilon}{\varepsilon-1} \frac{w+\tau b}{P}=\phi\left(x_{0}\right) .
$$

Since this is independent of $x$, the value-weighted marginal productivity is equalized across the plants. The driving force for such equalization is perfect mobility of the resource. Although a low- $x$ variety has a lower productivity than a high- $x$ variety, a smaller amount of a low- $x$ variety is produced. Since consumers value all varieties and the marginal utility of a variety is diminishing, a low- $x$ variety generates a higher marginal utility (and hence a higher price) than a high- $x$ variety. Precisely, productivity $e^{x}$ is weighted by $[Q / q(x)]^{1 / \varepsilon}$ in the formula above, and the ratio $Q / q(x)$ is decreasing in $x$. Weighted by the marginal value in $Q$, productivity is the same in all varieties.

When low- $x$ plants shut down under the emission standard and the resource is reallocated to high- $x$ plants, output of the remaining plants increases. This higher quantity reduces the marginal contribution of each remaining variety to $Q$, because this marginal contribution is diminishing. In the new equilibrium, the marginal contribution of the input is equalized at a lower level across the remaining plants. We can see this loss in productivity by rewriting the average productivity equivalently as

$$
\frac{1}{L} \int_{x_{0}}^{\infty} \frac{\partial Q}{G^{\prime}(x) \partial \ell(x)}\left[\ell(x) G^{\prime}(x)\right] d x=\frac{\partial Q}{G^{\prime}(x) \partial \ell(x)} .
$$

As the marginal productivity of each remaining variety (i.e., the right-hand side) falls, so does the average productivity.

Now it is easy to understand the results $P_{\tau}<P_{s}$ and $Q_{\tau}>Q_{s}$. The price level is a constant markup of the average effective marginal cost, $(w+\tau b) / \phi$. Since the average productivity is higher under the tax than under the standard, the average effective marginal cost is lower and, hence, the price level is lower under the tax. Moreover, higher 
productivity under the tax directly translates into higher output of the composite, because total input in the dirty-goods sector is the same under the two policies. It is worthwhile emphasizing that the tax has such superiority over the standard for all values of market power, i.e., for all $\varepsilon>1$.

\section{Equilibrium and Policy Analysis with Abatement}

Now let us introduce an abatement technology through (4). The main purpose is to uncover the interaction between productivity dispersion and abatement. By analyzing this interaction, we bring out the role of market power in policy evaluation that has been emphasized by the literature originated from Buchanan (1969) (see the introduction) and show how this role is limited by productivity dispersion.

\subsection{Equilibrium characterization under each policy}

A household's decisions are the same as those in subsection 3.1. A plant's decisions need to be modified. Consider the tax first. With the input in production, $\ell(x)$, and the input in abatement, $a(x)$, profit of a plant $x$ is

$$
\pi(x)=P Q^{\frac{1}{\varepsilon}}\left[e^{x} \ell(x)\right]^{1-\frac{1}{\varepsilon}}-w \ell(x)-\tau b\left(1+\frac{a(x)}{\ell(x)}\right)^{-\frac{1}{\gamma}} \ell(x)-w a(x),
$$

where we have substituted the demand function, (5), and the emission process, (4). The plant chooses the abatement level, $a(x)$, and the input, $\ell(x)$, to maximize profit. It is easy to verify that the plant's optimal choices $\operatorname{are}^{12}$

$$
\begin{aligned}
& a(x)=\ell(x)\left[\left(\frac{\tau b}{\gamma w}\right)^{\frac{\gamma}{\gamma+1}}-1\right], \\
& \ell(x)=Q e^{(\varepsilon-1) x}\left[\frac{(\varepsilon-1) P}{\varepsilon k}\right]^{\varepsilon},
\end{aligned}
$$

where

$$
k \equiv w(\gamma+1)\left(\frac{\tau b}{\gamma w}\right)^{\frac{\gamma}{\gamma+1}}
$$

\footnotetext{
${ }^{12}$ To avoid unnecessary complications, we allow the choice $a$ to be negative as well as positive, provided $1+\frac{a}{\ell} \geq 0$. The interpretation of a choice $a<0$ is that the plant uses a production technology that produces more emission than the production technology in the baseline model.
} 
By comparing (17) with its counterpart without the abatement choice, (8), we can interpret $k$ as the plant's marginal cost which incorporates the marginal cost of the inputs in production and abatement, and the tax. Adjusted for the plant's productivity, the plant's effective marginal cost is $k e^{-x}$. The optimal choices above induce the following levels of output, price, and emission under the tax:

$$
q(x)=Q e^{\varepsilon x}\left[\frac{(\varepsilon-1) P}{\varepsilon k}\right]^{\varepsilon}, \quad p(x)=\frac{\varepsilon k e^{-x}}{\varepsilon-1}, \quad m(x)=b\left(\frac{\tau b}{\gamma w}\right)^{\frac{-1}{\gamma+1}} \ell(x) .
$$

As in the baseline model, the price of a plant's product is a constant markup over the plant's effective marginal cost. Moreover, $\pi(x)>0$ for all $x$, and so all plants operate under the tax. That is, the set of productivity levels observed in the economy is $X=[\underline{x}, \infty)$.

Next, consider the emission standard. Profit of a plant $x$ is

$$
\pi(x)=P Q^{\frac{1}{\varepsilon}}\left[e^{x} \ell(x)\right]^{1-\frac{1}{\varepsilon}}-w \ell(x)-w a(x) .
$$

The standard imposes the constraint, $m(x) / q(x) \leq s$. With the emission process, (4), we can rewrite this constraint as $a(x) \geq \ell(x)\left[\left(\frac{b}{s}\right)^{\gamma} e^{-\gamma x}-1\right]$. Maximizing profit under this constraint, a plant $x$ 's optimal choices of $a$ and $\ell$ are:

$$
\begin{gathered}
a(x)=\ell(x)\left[\left(\frac{b}{s}\right)^{\gamma} e^{-\gamma x}-1\right], \\
\ell(x)=Q e^{[\varepsilon(\gamma+1)-1] x}\left[\frac{(\varepsilon-1) P}{\varepsilon w}\left(\frac{s}{b}\right)^{\gamma}\right]^{\varepsilon} .
\end{gathered}
$$

Under the standard, a plant-x's output, price and emission are

$$
\begin{gathered}
q(x)=Q e^{\varepsilon(\gamma+1) x}\left[\frac{(\varepsilon-1) P}{\varepsilon w}\left(\frac{s}{b}\right)^{\gamma}\right]^{\varepsilon}, \\
p(x)=\frac{\varepsilon e^{-(\gamma+1) x}}{\varepsilon-1} w\left(\frac{b}{s}\right)^{\gamma}, \quad m(x)=s q(x) .
\end{gathered}
$$

The plant's marginal cost is $w\left(\frac{b}{s}\right)^{\gamma} e^{-\gamma x}$, which includes the price of the input and the marginal cost of abatement. The effective marginal cost is $w\left(\frac{b}{s}\right)^{\gamma} e^{-(\gamma+1) x}$.

In contrast to the model without abatement, the model with abatement implies that every plant operates. To meet the standard, a plant spends enough in abatement and cuts 
production sufficiently. Since all varieties are produced under both policies, we will omit the notation for the interval $[\underline{x}, \infty)$ over which the integrals are computed.

The aggregate input in production, $L$, and the average productivity, $\phi$, are computed similarly to the baseline model. The aggregate level of abatement is $A=\int a(x) d G(x)$. An equilibrium can be defined by incorporating abatement into the definition in the baseline model. The following lemma determines the equilibrium (see Appendix B for a proof):

Lemma 5 Assume that the abatement technology (4) is available. Under either policy, there is a unique equilibrium, where $L=Q / \phi$ and $Q$ is determined by (6). Under the emission tax, $\phi=\phi(\underline{x})$, where the function $\phi($.$) is given by (12), and other aggregate$ quantities and prices are as follows:

$$
\begin{aligned}
& \tau=\frac{\gamma w}{b}\left(\frac{b L}{M}\right)^{\gamma+1}, \quad P=\frac{\varepsilon w(\gamma+1)}{(\varepsilon-1) \phi}\left(\frac{b Q}{M \phi}\right)^{\gamma}, \\
& A=L\left[\left(\frac{b L}{M}\right)^{\gamma}-1\right], \quad c=w-\frac{w Q}{\phi}\left(\frac{b Q}{M \phi}\right)^{\gamma} .
\end{aligned}
$$

Under the emission standard, aggregate quantities and prices are:

$$
\begin{gathered}
\phi=\left[\int e^{(\gamma+1)(\varepsilon-1) x} d G(x)\right]^{\frac{\varepsilon}{\varepsilon-1}} / \int e^{[\varepsilon(\gamma+1)-1] x} d G(x), \\
s=\frac{M}{L} \frac{\int e^{[\varepsilon(\gamma+1)-1] x} d G(x)}{\int e^{\varepsilon(\gamma+1) x} d G(x)}, \quad P=\frac{\varepsilon w \lambda}{(\varepsilon-1) \phi}\left(\frac{b Q}{M \phi}\right)^{\gamma}, \\
A=L\left[\left(\frac{b L}{M}\right)^{\gamma} \lambda-1\right], \quad c=w-\frac{w Q}{\phi}\left(\frac{b Q}{M \phi}\right)^{\gamma} \lambda,
\end{gathered}
$$

where

$$
\lambda \equiv \frac{\left[\int e^{(\varepsilon-1)(\gamma+1) x} d G(x)\right]\left[\int e^{\varepsilon(\gamma+1) x} d G(x)\right]^{\gamma}}{\left[\int e^{[\varepsilon(\gamma+1)-1] x} d G(x)\right]^{\gamma+1}} .
$$

\subsection{Comparing the two policies}

With the abatement choice, aggregate quantities depend on the distribution of productivity in a complicated way as shown in Lemma 5. To gain insight, let us assume a specific distribution function of $x$ and a specific utility function in this subsection. We will consider more general forms of these functions in section 5 . 
The specific distribution function (cdf) of $x$ is exponential:

$$
G(x)=1-e^{-(x-\underline{x}) / \delta}, \quad \delta \in(0, \bar{\delta}),
$$

where $\bar{\delta}=\min \left\{\frac{1}{2}, \frac{1}{\varepsilon(\gamma+1)}\right\}$. This distribution implies that productivity $z=e^{x}$ is distributed according to the Pareto distribution: $G_{z}(z)=1-\left(\frac{e^{\underline{x}}}{z}\right)^{1 / \delta}$. The restriction $\delta<1 /[\varepsilon(\gamma+1)]$ is required for $\lambda$ in (29) to be finite, while the restriction $\delta<1 / 2$ is required for the variance of $e^{x}$ to be finite. Under the restriction $\delta<1 / 2$, the mean of $e^{x}$ is $\frac{e^{\underline{x}}}{1-\delta}$ and the variance is $\frac{\delta^{2} e^{2 \underline{x}}}{(1-2 \delta)(1-\delta)^{2}}$. If we fix the mean of $e^{x}$ at any arbitrary level $\bar{z}>0$ by setting $\underline{x}=\ln [(1-\delta) \bar{z}]$, then the variance of $e^{x}$ is $\frac{(\delta \bar{z})^{2}}{1-2 \delta}$. Since this variance is increasing in $\delta$, we refer to $\delta$ as productivity dispersion among the plants.

The utility function is assumed to be

$$
u(c, Q, M)=U(c+v(M) Q), \text { with } U^{\prime}>0, U^{\prime \prime}<0, v>0, v^{\prime}<0 .
$$

With this utility function, the marginal rate of substitution between the dirty-goods composite and the clean good is $u_{2} / u_{1}=v(M)$. For any given emission target $M$, the equilibrium price of the dirty-goods composite is a constant $P=v(M)$. With (31), we can solve equilibrium $Q$ explicitly from (6) as

$$
Q= \begin{cases}\frac{M}{b}\left[\frac{(\varepsilon-1) v(M)}{\varepsilon w} \frac{\left(\phi_{\tau}\right)^{\gamma+1}}{\gamma+1}\right]^{1 / \gamma}, & \text { with the tax } \\ \frac{M}{b}\left[\frac{(\varepsilon-1) v(M)}{\varepsilon w} \frac{\left(\phi_{s}\right)^{\gamma+1}}{\lambda}\right]^{1 / \gamma}, & \text { with the standard. }\end{cases}
$$

For any given emission target, the equilibrium value of $Q$ differs under the two policies in two aspects. One is the difference in the average productivity. The other is that there is a constant $(\gamma+1)$ under the tax, while the corresponding constant is $\lambda$ under the standard.

Add the subscript $\tau$ to the variables under the tax and the subscript $s$ under the standard. In Appendix C, we prove the following proposition:

Proposition 6 Assume that the abatement technology in (4) is available and that productivity is distributed according to (30). Then, $\phi_{s}<\phi_{\tau}$. Define

$$
\varepsilon_{0}=\frac{1}{\gamma}\left[(\gamma+1)^{(\gamma+1) / \gamma}-1\right] \quad(>1) .
$$


With the utility function (31), $P_{\tau}=P_{s}=v(M)$, and the following results hold: (i) $\left(\frac{Q}{L+A}\right)_{s}<\left(\frac{Q}{L+A}\right)_{\tau}$; (ii) If $\varepsilon \geq \varepsilon_{0}$, then $u_{s}<u_{\tau}$ for all $\delta$; if $\varepsilon<\varepsilon_{0}$, then there exists $\delta_{0}(\varepsilon) \in(0, \bar{\delta})$ such that $u_{s}>u_{\tau}$ iff $\delta<\delta_{0}(\varepsilon)$; (iii) $u_{s}>u_{\tau} \Longrightarrow Q_{s}>Q_{\tau} \Longrightarrow L_{s}>L_{\tau} \Longrightarrow$ $A_{s} / L_{s}>A_{\tau} / L_{\tau}$ and $c_{s}<c_{\tau}$; (iv) The emission target that maximizes utility is the same under the two policies and is given by $M^{*}=\arg \max M[v(M)]^{(1+\gamma) / \gamma}$.

Introducing the abatement choice does not change the ranking of the two policies in the average productivity. Measured as $\phi$, the average productivity is still higher under the tax than under the standard. In contrast to the baseline model, however, the standard induces lower average productivity than the tax not by shutting down low- $x$ plants, but by reducing low- $x$ plants' input and shifting it to high- $x$ plants. This shift occurs because a low- $x$ plant must have a higher abatement intensity than a high- $x$ plant in order to meet the standard (see (20)). Since the marginal contribution of each variety to the composite is diminishing, this shift of the production from low-output varieties to high-output varieties reduces the average contribution of the input to the composite. In contrast, the tax does not induce this shift of the input, because the the abatement intensity is constant across the plants under the tax. Again, the average productivity is lower under the standard. Note that this result does not rely on the particular utility function (31).

The measure $\phi$ counts only the input in production of the dirty goods but not the input in abatement. If the input in abatement is also counted, total input in the dirtygoods sector is $(L+A)$, and the overall productivity in the sector is $Q /(L+A)$. Part (i) of Proposition 7 says that, when the utility function has the form in (31), the overall productivity is also higher under the tax than under the standard. ${ }^{13}$

Introducing the abatement choice can modify the ranking of the two policies in other aspects, including the ranking in welfare. As stated in (ii) of Proposition 7, the standard yields higher welfare than the tax if productivity dispersion is sufficiently small and if market power of each variety is sufficiently strong. Part (iii) provides a list of comparisons

\footnotetext{
${ }^{13} \mathrm{As}$ in the baseline model, one can compute the effective marginal productivity of the input in each plant $x$ as $\frac{1}{G^{\prime}(x)} \partial Q / \partial[\ell(x)+a(x)]$ and verify that it is equal to $Q /(L+A)$.
} 
between the two policies. In particular, for the standard to yield higher welfare than the tax, the standard must induce higher output of the dirty-goods composite, a higher input into production of dirty goods, a higher intensity (and level) of abatement, and lower consumption of the clean good. Part (iv) says that the optimal emission target is the same under the two policies. An implication is that the same ranking described in parts (i) (iii) is valid if the emission target is chosen optimally under each policy.

In the next two subsections, we will explain why these modifications occur. Before doing so, it is useful to emphasize that market power affects the welfare ranking between the two policies only when productivity dispersion is small. Even in the limit case $\varepsilon \rightarrow 1$, where a plant's market power is the largest, the critical level on productivity dispersion, $\delta_{0}(\varepsilon)$, satisfies $\delta_{0}(\varepsilon)<\bar{\delta}$. This means that if productivity is sufficiently dispersed in the sense $\delta>\delta_{0}(1)$, then the tax is superior to the standard for all values of market power even when abatement is available. In contrast, the literature that focuses on market power but abstracts from productivity dispersion shows that the standard is superior to the tax when market power is sufficiently large (see the introduction for references).

\subsection{Why is the standard possibly better than the tax?}

The answer to this question must lie in the response of abatement to the two policies, because the standard is always inferior to the tax in the economy without an abatement technology. To explain the role of abatement clearly, it is useful to isolate this role by abstracting from productivity dispersion. Accordingly, in this subsection we assume that all plants have the same value of $x$. In this economy, the following proposition states the ranking between the two policies (see Appendix B for a proof): ${ }^{14}$

Proposition 7 When there is no productivity dispersion, the equilibrium with the abatement choice yields $\phi_{s}=\phi_{\tau}, \lambda=1, Q_{s}>Q_{\tau}, L_{s}>L_{\tau}, A_{s} / L_{s}>A_{\tau} / L_{\tau}$ and $c_{s}<c_{\tau}$ for any given emission target $M$. Moreover, a sufficient condition for $u_{s}>u_{\tau}$ is $\varepsilon \leq 1+\frac{1}{\gamma}$. On the other hand, if $\varepsilon$ is sufficiently large, then $u_{s}<u_{\tau}$.

\footnotetext{
${ }^{14}$ The results in Proposition 7 hold for general utility functions, not just for that in (31).
} 
When all plants are homogeneous in productivity, the average productivity is the same under both policies, and the distribution of relative prices between the varieties is degenerate. In this economy, the two policies both increase the effective marginal cost of production of the dirty goods, exert an upward pressure on the price, and hence reduce output of the dirty goods. The main difference between the two policies is that the standard increases the effective marginal cost by less than the tax does, as explained below. As a result, the standard generates higher input and output in the dirty-goods sector than the tax does. Moreover, because the emission target is the same under the two policies and output of the dirty goods is higher under the standard, the emission intensity is lower under the standard than under the tax. To generate a lower emission intensity, the standard must induce a higher abatement intensity than the tax does, as stated in Proposition 7. Because the standard induces more resources to be used in the dirty-goods sector, a lower quantity of the clean good is produced under the standard than under the tax.

The welfare ranking between the two policies depends on the relative change in the two types of consumption. This is where market power plays a role. When market power of each variety in the dirty-goods sector is sufficiently strong in the sense that $\varepsilon \leq 1+\frac{1}{\gamma}$, the markup of price on the marginal cost is high. In this case, even a small difference in the marginal cost can translate into a large difference in price and, hence, in output. The additional quantity of the dirty-goods composite produced under the standard relative to the tax can be so large that it outweighs the shortfall in the clean good. On the other hand, if market power is weak, the standard yields lower welfare than the tax, because the additional quantity of the dirty goods produced under the standard is too small to compensate for the shortfall in the clean good. Note that this role of market power in policy evaluation is to determine the balance between the two sets of goods and, as such, it is a feature of the general equilibrium of our model. This role is different from the one in a partial equilibrium which is to determine which policy yields higher output of the dirty goods. In the economy with abatement and homogeneous productivity, output of the dirty goods is always higher under the standard than under the tax. 
We now explain why the standard increases the marginal cost by less than the tax does when the plants are homogeneous in productivity. A plant's effective marginal cost consists of the direct and the indirect marginal cost. The direct marginal cost $w$ is the same under the two policies, but the indirect marginal cost is different. To meet the standard on the emission intensity, a plant keeps the abatement intensity at a constant ratio that is a function of the standard. When the input increases, the required level of abatement increases at this ratio, which is the indirect marginal cost. Under the tax, it is also optimal for a plant to keep the abatement intensity at a constant ratio that is a function of the policy (tax). And so the abatement cost increases according to this ratio as the input in production increases. However, this is not the only indirect marginal cost under the tax. Even when the abatement intensity is kept constant, an increase in the input in production increases emission (see (4)), which is taxed. This additional cost under the tax induces a plant to restrict output by more than under the standard.

To support the above explanation, recall that a plant's effective marginal cost of production is $w(\gamma+1)\left(\frac{\tau b}{\gamma w}\right)^{\frac{\gamma}{\gamma+1}} e^{-x}$ under the tax and $w\left(\frac{b}{s}\right)^{\gamma} e^{-(\gamma+1) x}$ under the standard. When there is no dispersion in productivity, each plant's input, abatement, and emission are equal to their industry average. That is, $\ell(x)=L, a(x)=A$ and $m(x)=M$. From the equation $m(x)=M$, we can solve the policy level $(s$ or $\tau$ ) that is required to meet the target $M$. At this policy level, the effective marginal cost of production is $w(\gamma+1)\left(\frac{b L}{M}\right)^{\gamma} e^{-x}$ under the tax and $w\left(\frac{b L}{M}\right)^{\gamma} e^{-x}$ under the standard. Clearly, the former is higher than the latter by a factor $(\gamma+1)$. Since the price level is $\varepsilon /(\varepsilon-1)$ times the effective marginal cost, it is also higher under the tax than under the standard by a factor $(\gamma+1) .{ }^{15}$

\subsection{Interaction between productivity dispersion and abatement}

Let us now return to the economy with productivity dispersion in order to examine the interaction between productivity dispersion and abatement.

Productivity heterogeneity can reverse the above cost advantage of the standard over

\footnotetext{
${ }^{15}$ It can be verified that, with homogeneous productivity, the effective marginal cost of production is higher under the tax than under the standard even for a general emission process, $m(x)=\mu(\ell(x), a(x))$, where $\mu_{1}>0$ and $\mu_{2}<0$.
} 
the tax. First of all, with productivity dispersion, the average productivity in the dirtygoods sector is lower under the standard than under the tax, as we explained earlier. A lower average productivity adds upward pressure on the price. Moreover, the standard tilts abatement to the plants with low productivity and high cost, thus pushing up the marginal cost by more at plants with high prices than at plants with low prices. This change in the distribution of relative prices also adds upward pressure on the price index under the standard, which is absent under the tax.

To explain why the standard changes the distribution of relative prices, note that low- $x$ plants must have a higher abatement intensity than high- $x$ plants do in order to meet the same standard (see the explanation after Proposition 6). Thus, the standard increases low- $x$ plants' marginal costs and prices by more than high- $x$ plants'. Under monopolistic competition, low- $x$ plants produce less and charge higher prices than high- $x$ plants do. Imposing the standard increases high prices by a larger proportion than low prices, thus tilting the distribution of relative prices toward high prices. This effect is reflected by an increase in $\lambda$, defined in (29), which increases $P$. In contrast, the tax induces all plants to spend the same proportion in abatement relative to their input in production. Thus, prices of all varieties increase by the same proportion under the tax, leaving the relative price between any two plants unchanged.

Overall, whether the standard or the tax creates higher or lower upward pressure on the price index depends on the extent of productivity dispersion. When productivity dispersion is sufficiently small, i.e., when $\delta$ is small, the two new forces above on the price index created by the standard are small. In this case, the standard has lower upward pressure on the price index than the tax. When productivity dispersion is sufficiently high, the two new forces above on the price index dominate the force in the economy with homogeneous plants. In this case, the standard has higher upward pressure on the price index than the tax.

Differences between the two policies' pressure on the price index translate into differences in the quantity of the dirty-goods composite which, in turn, affect the welfare ranking of the two policies as stated in (ii) of Proposition 6. With the utility function (31), the 
equilibrium price is fixed by the target on aggregate emission as $p=v(M)$. Any upward pressure on the price index must be absorbed by a fall in output of the dirty-goods composite in order to restore the equilibrium. Thus, when productivity dispersion is small, the standard induces a higher quantity of the dirty-goods composite than the tax does. If market power is also sufficiently high, this higher quantity of dirty goods dominates the lower quantity of the clean good, as we explained in the previous subsection, and welfare is higher under the standard than under the tax. On the other hand, if productivity dispersion is high, the standard induces much lower average productivity and a much lower quantity of the dirty goods than the tax does, in which case the tax dominates the standard in welfare. With sufficiently high dispersion in productivity, this superiority of the tax holds regardless of the value of market power. ${ }^{16}$

Let us turn to the remaining parts of Proposition 6. Part (iii) is easy to understand. First, for utility to be higher under the standard than under the tax, the standard must generate less pressure on the price index of the dirty-goods composite, in which case the composite decreases by less under the standard than under the tax. Second, since productivity is lower under the standard than under the tax, the input in the dirty-goods sector must be higher under the standard in order to produce a higher composite of the dirty goods than under the tax. Third, with (4), abatement is proportional to the input in production. Thus, a higher input in the production of the dirty-goods composite also calls for a higher level of abatement. Finally, when the input in the dirty-goods sector is higher under the standard, the input in the clean-good sector is lower, which contributes to lower consumption of the clean good under the standard than under the tax.

Part (iv) of Proposition 6 describes the optimal emission target, i.e., the target that maximizes the representative household's utility. With (31), the optimal level of $c$ is proportional to $v Q$. Since $v Q$ depends on $M$ only through the term $M v^{(1+\gamma) / \gamma}$, so does the

\footnotetext{
${ }^{16}$ Note that the effectiveness of the abatement technology, $1 / \gamma$, also plays a role since the critical levels $\varepsilon_{0}$ and $\delta_{0}$ in Proposition 6 depend on $\gamma$. In the limit $\gamma \rightarrow 0$, even a tiny amount of input in abatement can reduce emission to zero. In this case, the two policies are equivalent. In the opposite limit $\gamma \rightarrow \infty$, the abatement is not effective at all, and the model approaches the baseline model where the tax induces higher welfare than the standard.
} 
utility level. The optimal target maximizes this term regardless of which policy is used to implement the target. This result implies that parts (i) - (iii) of Proposition 6 continue to hold when the emission target is set to the optimal level under each policy.

\section{Robustness of the Results}

Proposition 6 utilized the particular distribution function, (30), and the utility function, (31). These functional forms enabled us to solve the equilibrium explicitly. However, the main results hold more generally, as we illustrate in this section.

\subsection{The utility function}

Consider first the following generalization of the utility function:

$$
u(c, Q, M)=\left\{\alpha c^{\rho}+(1-\alpha)[v(M) Q]^{\rho}\right\}^{1 / \rho}, \alpha, \rho \in[0,1], v>0, \text { and } v^{\prime}<0
$$

The restriction $\rho \geq 0$ is imposed to satisfy Assumption 1 . The case $\rho=1$ corresponds to (31). The case $\rho=0$ is also analytically tractable and the results are the same as in Proposition 6 after a modification of $\varepsilon_{0}$. For any $\rho \in[0,1)$, the relative price of the dirtygoods composite to the clean good is endogenous, in contrast to the case $\rho=1$ where the price is fixed by the emission target. We use numerical examples to illustrate the results for $\rho \in(0,1)$. Let $v(M)=M^{-\kappa}$ and fix some of the parameters as follows:

$$
\alpha=0.6, \gamma=0.2, b=3, w=1, \underline{x}=\ln (1-\delta), \kappa=0.5
$$

The chosen value $\underline{x}$ implies that the mean of $e^{x}$ is 1 and the variance is $\frac{\delta^{2}}{1-2 \delta}$. We explore different values of $(\delta, \varepsilon, \rho)$. For each $\rho \in[0,1]$, we find the region of $(\delta, \varepsilon)$ in which the standard yields higher welfare than the tax.

Figure 1 depicts two sets of curves in the $(\delta, \varepsilon)$ plane. One is the curve below which the values of $(\delta, \varepsilon)$ are feasible. The other is the curve along which the two policies yield the same welfare, and is drawn for $\rho=0.1,0.5$ and 0.8 , respectively. The standard yields higher welfare than the tax if and only if the values of $(\delta, \varepsilon)$ lie below this curve. For all three values of $\rho$, the standard dominates the tax in welfare if and only if $\delta$ and $\varepsilon$ are 
small. This result is consistent with Proposition 6. In addition, when the elasticity of substitution between the clean and the dirty goods increases, i.e., when $\rho$ increases, the curve moves slightly upward for intermediate values of $\delta$, but downward for small values of $\delta$. This indicates that the more substitutable the clean and dirty goods are, the larger the parameter region in which the standard dominates the tax.

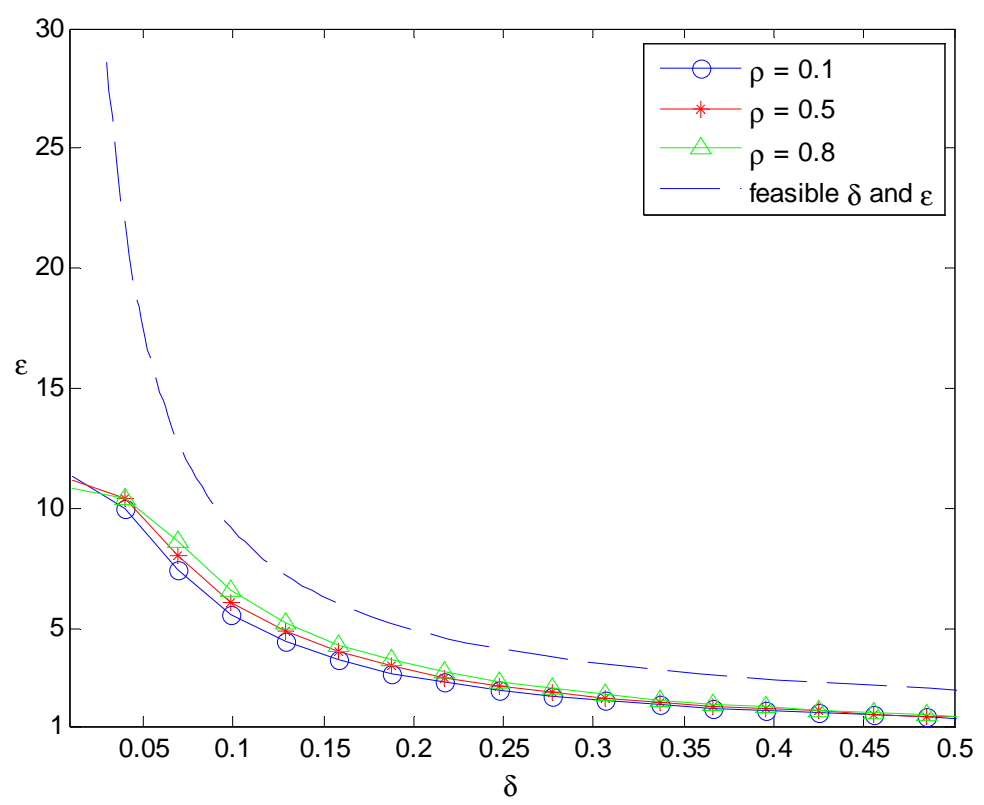

Figure 1. The region of $(\delta, \varepsilon)$ in which the standard dominates the tax

\subsection{The distribution function of productivity}

Now let us return to the utility function (31) but consider a different distribution of productivity. The density function of the distribution of productivity $z\left(=e^{x}\right)$ is given by

$$
G_{z}^{\prime}(z)= \begin{cases}\frac{\beta}{|\delta|}(z-\underline{z})^{1 / \delta-1} e^{-\beta(z-\underline{z})^{1 / \delta} d z,}, & \text { if } z>\underline{z} \\ 0, & \text { otherwise. }\end{cases}
$$

This distribution is Weibull if $\delta>0$ and Frechet if $\delta<0$. We normalize $\underline{z}=0$ to simplify the algebra. The mean of productivity $z$ is $\beta^{-\delta} \Gamma(1+\delta)$, and the variance of $z$ is $\beta^{-2 \delta}\left[\Gamma(1+2 \delta)-[\Gamma(1+\delta)]^{2}\right]$, where $\Gamma$ is the gamma function. If we choose $\beta$ to fix the mean of $z$ at any arbitrary level $\bar{z}$, then the variance of $z$ is $\bar{z}^{2}\left[\frac{\Gamma(1+2 \delta)}{[\Gamma(1+\delta)]^{2}}-1\right]$. Since this 
variance is increasing in $|\delta|$, we refer to $|\delta|$ as a measure of productivity dispersion among the plants. We restrict $\delta>-1 / 2$ for the variance to be bounded and restrict $\delta>-\frac{1}{\varepsilon(\gamma+1)}$ for $\lambda$ (defined in (29)) to exist.

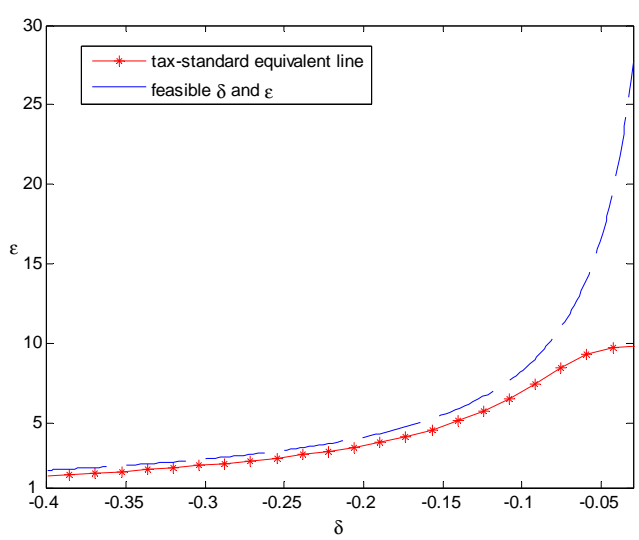

Frechet distribution

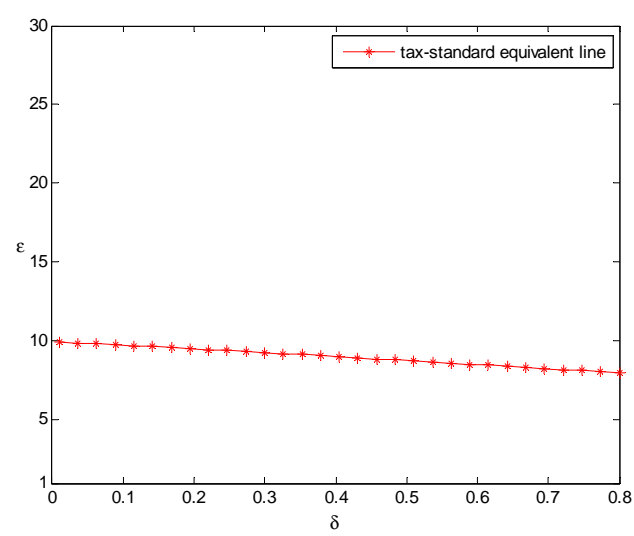

Weibull distribution

Figure 2. The region of $(\delta, \varepsilon)$ in which the standard dominates the tax

With (33), the average productivity under the two policies is, respectively:

$$
\phi_{\tau}=\beta^{-\delta}[\Gamma(1+\delta(\varepsilon-1))]^{\frac{1}{\varepsilon-1}}, \quad \phi_{s}=\frac{\beta^{-\delta}[\Gamma(1+\delta(\gamma+1)(\varepsilon-1))]^{\frac{\varepsilon}{\varepsilon-1}}}{\Gamma(1+\delta(\varepsilon(\gamma+1)-1))} .
$$

The term $\lambda$ defined in (29) is equal to

$$
\lambda=\frac{\Gamma(1+\delta(\gamma+1)(\varepsilon-1))[\Gamma(1+\delta \varepsilon(\gamma+1))]^{\gamma}}{[\Gamma(1+\delta(\varepsilon(\gamma+1)-1))]^{\gamma+1}} .
$$

Using (31), welfare is higher under the standard than under the tax if and only if

$$
\left(\frac{\phi_{s}}{\phi_{\tau}}\right)^{(\gamma+1)}\left(\frac{\gamma+1}{\varepsilon \gamma+1}\right)^{\gamma} \frac{\gamma+1}{\lambda}>1
$$

The condition (34) depends on four parameters: $\gamma, \delta, \varepsilon$, and $\beta$. We take $\gamma=0.2$ as in the previous subsection and choose $\beta$ to normalize the mean of productivity, $\beta^{-\delta} \Gamma(1+\delta)$, to 1 . The numerical results are depicted in Figure 2, where the left panel is for the Frechet distribution and the right panel for the Weibull distribution. The curve labeled "taxstandard equivalent line" contains the combinations of $\delta$ and $\varepsilon$ with which the two policies yield the same welfare. In the region below this line, the standard yields higher welfare 
than the tax. This region is non-empty for both the Frechet and the Weibull distribution. Thus, the standard yields higher welfare than the tax if and only if productivity dispersion is small (i.e., if $|\delta|$ small) and market power is strong (i.e., if $\varepsilon$ is small). Therefore, the main qualitative result in Proposition 6 also holds under the distribution function (33).

\section{Conclusion}

We have evaluated an emission tax against a nontradable emission standard in an economy with rich dispersion in productivity among a continuum of monopolistically competitive firms. We have found that productivity dispersion increases the advantage of the tax over the standard as the better policy. If no technology is available for emission abatement, then the tax induces higher average productivity, a lower average price, higher output and higher welfare than the standard. If an abatement technology is available, the standard can yield higher welfare than the tax if firms have both sufficiently similar productivity and sufficiently large market power. Moreover, the standard changes relative prices between firms' products by tilting abatement toward high-cost and low-productivity firms, which the tax does not do. When productivity dispersion exceeds a critical level, the tax is again superior to the standard for all values of market power. Therefore, productivity dispersion and its interaction with abatement choices are important for evaluating marketbased versus non market-based environmental policies.

One appealing feature of our model is that it can be integrated relatively easily with other fields of economics for a comprehensive study of environmental issues. First, it is straightforward to embed our model into a standard dynamic macro model with an infinite horizon and allow emission to accumulate and decumulate over time. Second, it is interesting to allow for entry and exit of the plants and calibrate the dynamic model to quantify the cost of reaching an environmental target. Li and Sun (2009) have made an attempt on such quantitative exercises. Finally, given the common emphasis on productivity dispersion by our model and the Eaton-Kortum-Melitz trade model, it is fruitful to study how environmental and trade policies should be coordinated. 


\section{Appendix}

\section{A Proofs of Lemma 3 and Proposition 4}

We establish Lemma 3 first. Under the tax, (14) can be written as

$$
R\left(w-w \frac{\bar{M}}{b}, \phi(\underline{x}) \frac{\bar{M}}{b_{0}}, \bar{M}\right)=P(\tau, \phi(\underline{x})) .
$$

For any given $\bar{M}$, the left-hand side of the above equation is independent of $\tau$ and the righthand side is an increasing function of $\tau$. Thus, there exists a unique level of $\tau$, denoted $\tau(\bar{M})$, that solves the above equation. With this level of the tax, other equilibrium variables are uniquely determined as in the main text. Moreover, the target $\bar{M}$ is binding if and only if $\tau(\bar{M})>0$. The latter requirement is equivalent to the condition that the left-hand side of the above equation is strictly greater than $P(0, \phi(\underline{x}))$. Since the left-hand side of the above equation is a strictly decreasing function of $\bar{M}$ (see Assumption 1), the target is binding if and only if $\bar{M}<M_{\max }$. It is easy to see that $\tau^{\prime}(\bar{M})<0$ and $\tau\left(M_{\max }\right)=0$.

Under the emission standard, $x_{0}=\ln (b / s)$, and (14) can be written as

$$
\phi R\left(w-w \frac{\bar{M}}{b}, \phi \frac{\bar{M}}{b}, \bar{M}\right)=\frac{\varepsilon}{\varepsilon-1} w,
$$

where $\phi=\phi(\ln (b / s))$. For any given $\bar{M}$, the assumption on $q R$ imposed in Lemma 3 ensures that the left-hand side of the above equation is a strictly increasing function of $\phi$ and reaches 0 at $\phi=0$. There is a unique level of $\phi$, denoted $\phi_{s}(\bar{M})$, that solves the above equation. The implied standard can be calculated from $\phi_{s}(\bar{M})=\phi(\ln (b / s(\bar{M})))$ and other equilibrium variables can be uniquely determined as in the main text. Moreover, the target $\bar{M}$ is binding iff $\ln (b / s(\bar{M}))>\underline{x}$, i.e., iff $\phi_{s}(\bar{M})<\phi(\underline{x})$. This requirement is equivalent to $\bar{M}<M_{\max }$. Furthermore, it is easy to verify that $\phi_{s}^{\prime}(\bar{M})>0$ and $\phi_{s}\left(M_{\max }\right)=\phi(\underline{x})$. Hence, $s^{\prime}(\bar{M})>0$ and $s\left(M_{\max }\right)=b e^{-\underline{x}}$. This completes the proof of Lemma 3 .

Now we prove Proposition 4 by verifying statements (i)-(iii) in the proposition. Statement (i) is evident, since $L=\bar{M} / b$ and $c=w(1-L)$ under both policies. For statement (ii), the proof of Lemma 3 has already established $x_{0 \tau}=\underline{x}<\ln (b / s)=x_{0 s}$ for any binding 
target. Because $\phi\left(x_{0}\right)$ defined by (12) is a strictly decreasing function of $x_{0}$, then $\phi_{\tau}>\phi_{s}$. Since $Q=\phi \bar{M} / b$, then $Q_{\tau}>Q_{s}$. Recall that $P=R(c, Q, M)$ and that $R(c, Q, M)$ is a strictly decreasing function of $Q$. We have $P_{\tau}=R\left(c, Q_{\tau}, \bar{M}\right)<R\left(c, Q_{s}, \bar{M}\right)=P_{s}$. Finally, $u_{\tau}=u\left(c, Q_{\tau}, \bar{M}\right)>u\left(c, Q_{s}, \bar{M}\right)=u_{s}$. QED

\section{B Proofs of Lemma 5 and Proposition 7}

For Lemma 5, we only derive the formulas for the tax and prove that there exists a unique equilibrium. The derivation and the proof for the standard are similar and, hence, are omitted. Under the tax, we substitute $q(x)$ from (19) into (2) to compute $Q$ and aggregate $\ell(x)$ from (17) to compute $L$, which yields $\phi=Q / L=\phi(\underline{x})$, where $\phi($.$) is the function$ defined by (12). Aggregating $m(x)$ in (19), we have $M=b\left(\frac{\tau b}{\gamma w}\right)^{\frac{-1}{\gamma+1}} L$. Inverting this result yields $\tau$ as the function of $(L, M)$ in (24). Substituting $p(x)$ from (19) into (7), we obtain $P=\frac{\varepsilon k}{(\varepsilon-1) \phi}$, where $k$ is a function of $\tau$ given by (18). Substituting $\tau$, we obtain

$$
k=w(\gamma+1)\left(\frac{b L}{M}\right)^{\gamma}
$$

and, hence, $P$ is as in (24). Aggregating $a(x)$ in (16) and substituting $\tau$ from (24), we obtain $A$ as in (25). Since total input in the dirty-goods sector is $(L+A)$, consumption of the clean good is $c=w(1-L-A)$. Substituting $A$, we obtain $c$ as in (25). The quantity $Q$ is determined by (6). Proving that there exists a unique equilibrium amounts to proving that there is unique solution for $Q$. Substituting $P$ from (24), we have

$$
\frac{u_{2}(c(Q), Q, M)}{u_{1}(c(Q), Q, M)}=\frac{\varepsilon w(\gamma+1)}{(\varepsilon-1) \phi}\left(\frac{b Q}{M \phi}\right)^{\gamma},
$$

where $c(Q)$ is given by (25). Under Assumption 1, the left-hand side of (36) is a strictly decreasing function of $Q$, while the right-hand side is a strictly increasing function of $Q$. With these features, it is easy to prove that there is a unique solution for $Q$ to (36). This completes the proof of Lemma 5.

For Proposition 7, we assume that the measure of plants is one without loss of generality. When all plants have the same productivity, $\ell(x)=L, a(x)=A, q(x)=Q$, and $m(x)=M$. 
It is evident that $\phi_{s}=\phi_{\tau}=e^{x}$ and $\lambda=1$. Substituting the pricing formulas in (24) and (27) into (6), we obtain:

$$
\frac{u_{2}(c, Q, M)}{u_{1}(c, Q, M)}=\frac{\sigma \varepsilon w}{(\varepsilon-1) \phi}\left(\frac{b Q}{M \phi}\right)^{\gamma},
$$

where $\sigma=\gamma+1$ under the tax and $\sigma=1$ under the standard. Under both policies,

$c=w-\frac{w Q}{\phi}\left(\frac{b Q}{M \phi}\right)^{\gamma}$ and $\phi=e^{x}$ in the above equation. Denote the solution to the above equation as $Q(\sigma)$. It is clear that $Q^{\prime}(\sigma)<0$. Thus, $Q_{s}>Q_{\tau}$. From (25), (28) and $L=Q / \phi$, it is clear that $L_{s}>L_{\tau}, A_{s} / L_{s}>A_{\tau} / L_{\tau}$, and $c_{s}<c_{\tau}$. Express the utility level as $\hat{u}(\sigma) \equiv u(c(\sigma), Q(\sigma), M)$. Then, $u_{s}>u_{\tau}$ iff $\hat{u}(1)>\hat{u}(\gamma+1)$. It can be verified that $\hat{u}^{\prime}(\sigma)<0$ iff $\sigma>(\gamma+1)(\varepsilon-1) / \varepsilon$. If $1 \geq(\gamma+1)(\varepsilon-1) / \varepsilon$, i.e., if $\varepsilon \leq 1+\frac{1}{\gamma}$, then $\hat{u}^{\prime}(\sigma)<0$ for all $\sigma>1$. In this case, $u_{\tau}=\hat{u}(\gamma+1)<\hat{u}(1)=u_{s}$. On the other hand, if $\varepsilon \rightarrow \infty$, then $\hat{u}^{\prime}(\sigma)>0$ for all $\sigma<\gamma+1$. In this case, $u_{\tau}=\hat{u}(\gamma+1)>\hat{u}(1)=u_{s}$. QED

\section{Proof of Proposition 6}

Using (30), we compute the average productivity as

$$
\phi= \begin{cases}{[1-\delta(\varepsilon-1)]^{\frac{-1}{\varepsilon-1}} e^{\underline{x}},} & \text { with the tax } \\ \frac{1+\delta-\delta \varepsilon(\gamma+1)}{[1-\delta(\varepsilon-1)(\gamma+1)]^{\frac{\varepsilon}{\varepsilon-1}}} e^{\underline{x}}, & \text { with the standard. }\end{cases}
$$

The statement $\phi_{s}<\phi_{\tau}$ is true if and only if

$$
\frac{\varepsilon}{\varepsilon-1} \ln [1-\delta(\varepsilon-1)(\gamma+1)]-\ln [1+\delta-\delta \varepsilon(\gamma+1)]-\frac{1}{\varepsilon-1} \ln [1-\delta(\varepsilon-1)]>0 .
$$

Temporarily denote the left-hand side as $L H S(\delta)$. Note that $L H S(0)=0$ and $L H S\left(\frac{1}{\varepsilon(\gamma+1)}\right)>$ 0 . Also, $L H S^{\prime}(\delta)$ has the same sign as the following expression:

$$
\frac{\delta \varepsilon \gamma(\gamma+1)}{[1-\delta(\varepsilon-1)(\gamma+1)][1+\delta-\delta \varepsilon(\gamma+1)]}+\frac{1}{[1-\delta(\varepsilon-1)]}
$$

Thus, $L H S^{\prime}(\delta)>0$. For all $\delta>0, L H S(\delta)>L H S(0)=0$.

With the utility function (31), it is clear that $P_{s}=P_{\tau}=v(M)$. For part (i) of the proposition, we can compute

$$
\frac{Q}{L+A}= \begin{cases}\frac{\varepsilon w}{(\varepsilon-1) v(M)}(\gamma+1), & \text { with the tax } \\ \frac{\varepsilon w(M)}{(\varepsilon-1) v(M)}, & \text { with the standard. }\end{cases}
$$


It is evident that $Q /(L+A)$ is higher under the tax than under the standard.

For other parts of the proposition, we substitute $G$ from (30) into (29) to compute

$$
\lambda=\frac{[1+\delta-\delta \varepsilon(\gamma+1)]^{\gamma+1}}{[1-\delta \varepsilon(\gamma+1)]^{\gamma}[1-\delta(\varepsilon-1)(\gamma+1)]}(>1) .
$$

For part (ii), we use (32) to solve for $L, A$ and $c$. Substituting $(c, Q)$ and $\lambda$ into (31), we know that $u_{s}>u_{\tau}$ iff $\frac{Q_{s}}{Q_{\tau}}>\frac{\varepsilon \gamma+1}{\gamma+1}$. Write the latter condition equivalently as follows:

$$
\begin{aligned}
& \frac{1}{\varepsilon-1} \ln [1-\delta(\varepsilon-1)]+\frac{\gamma}{\gamma+1} \ln [1-\delta \varepsilon(\gamma+1)] \\
& -\frac{\varepsilon \gamma+1}{(\varepsilon-1)(\gamma+1)} \ln [1-\delta(\varepsilon-1)(\gamma+1)]+\ln (\gamma+1)-\frac{\gamma}{\gamma+1} \ln (\varepsilon \gamma+1) \quad>0 .
\end{aligned}
$$

Temporarily denote the left-hand side as $\operatorname{LHS}(\delta)$. It is clear that $\operatorname{LHS}\left(\frac{1}{\varepsilon(\gamma+1)}\right)=-\infty$. Also, $\operatorname{LHS}(0)>0$ iff $\varepsilon<\varepsilon_{0}$, where $\varepsilon_{0}$ is defined in the proposition. Moreover, we can verify that

$$
L H S^{\prime}(\delta)=\frac{-\delta \gamma(\varepsilon \gamma+1)}{[1-\delta(\varepsilon-1)][1-\delta(\varepsilon-1)(\gamma+1)][1-\delta \varepsilon(\gamma+1)]}<0 .
$$

If $\varepsilon \geq \varepsilon_{0}$, then $\operatorname{LHS}(\delta)<\operatorname{LHS}(0) \leq 0$, in which case $u_{s}<u_{\tau}$ for all $\delta \in(0, \bar{\delta})$. If $\varepsilon<\varepsilon_{0}$, then $\operatorname{LHS}(0)>0$, in which case there exists $\delta_{0}(\varepsilon) \in(0, \bar{\delta})$ such that $u_{s}>u_{\tau}$ iff $\delta<\delta_{0}(\varepsilon)$. Moreover, using L'Hopital's rule, we can verify that $L H S(\bar{\delta})<0$ in the limit $\varepsilon \rightarrow 1$. This implies the strict inequality, $\delta_{0}(\varepsilon)<\bar{\delta}$, even in the limit $\varepsilon \rightarrow 1$.

For part (iii), recall that $u_{s}>u_{\tau}$ iff $\frac{Q_{s}}{Q_{\tau}}>\frac{\varepsilon \gamma+1}{\gamma+1}$. Because $\varepsilon>1$, then $u_{s}>u_{\tau}$ implies $Q_{s}>Q_{\tau}$. Similarly, since $L=Q / \phi$ and $\phi_{\tau}>\phi_{s}$, then $Q_{s}>Q_{\tau}$ implies $L_{s}>L_{\tau}$. To compare the aggregate level of abatement and consumption of the clean good under the two polices, recall that $A=L\left[\left(\frac{b L}{M}\right)^{\gamma}-1\right]$ and $c=w-w L\left(\frac{b L}{M}\right)^{\gamma}$ under the tax, while $A=L\left[\left(\frac{b L}{M}\right)^{\gamma} \lambda-1\right]$ and $c=w-w L\left(\frac{b L}{M}\right)^{\gamma} \lambda$ under the standard. Since $\lambda>1$, the inequality $L_{s}>L_{\tau}$ is sufficient for $A_{s} / L_{s}>A_{\tau} / L_{\tau}$ and $c_{s}<c_{\tau}$.

For part (iv), we can substitute equilibrium values of $(c, Q)$ into the utility function to verify that $u(c+v(M) Q)$ depends on $M$ entirely through the term $M[v(M)]^{(1+\gamma) / \gamma}$ and is increasing in this term. Then, part (iv) is evident. QED 


\section{References}

[1] Barnett, A.H., 1980. "The Pigouvian Tax Rule under Monopoly," American Economic Review 70, 1037-1041.

[2] Baumol, William J. and Wallace E. Oates, 1975. The Theory of Environmental Policy, Englewood Cliffs.

[3] Buchanan, James M., 1969. "External Diseconomies, Corrective Taxes, and Market Structure," American Economic Review 59, 174-177.

[4] Carraro, Carlo and Antoine Soubeyran, 1996. "Environmental Taxation, Market Share, and Profits in Oligopoly," in: Carraro, Carlo, Katsoulacos, Yannis and Anastasios Xepapadeas (eds.), Environmental Policy and Market Structure, Kluwer Academic Publishers, Dordrecht.

[5] Eaton, Jonathan and Samuel Kortum, 2002. "Technology, Geography, and Trade," Econometrica 70, 1741-1779.

[6] Fischer, Carolyn, 2011. "Market Power and Output-Based Refunding of Environmental Policy Revenues," Resource and Energy Economics 33, 212-230.

[7] Freeman, Jody and Charles D. Kolstad, 2007. Moving to Markets in Environmental Regulation: Lessons from Twenty Years of Experience, Oxford University Press.

[8] Gersbach, Hans and Till Requate, 2004. "Emission Taxes and Optimal Refunding Schemes," Journal of Public Economics 88, 713-725.

[9] Hepburn, Cameron, 2006. "Regulation by Prices, Quantities, or Both: A Review of Instrument Choice," Oxford Review of Economic Policy 22, 226-247.

[10] Holland, Stephen P., 2009. "Taxes and Trading versus Intensity Standards: SecondBest Environmental Policies with Incomplete Regulation (Leakage) or Market Power," NBER Working Paper 15262.

[11] IPCC (Intergovernmental Panel on Climate Change), 2007. "Fourth Assessment Report: Climate Change - Mitigation of Climate Change," Working Group III Report.

[12] Kelly, David L., 2005. "Price and Quantity Regulation in General Equilibrium," Journal of Economic Theory 125, 36-60. 
[13] Laffont, Jean Jacques, 1977. "More on Prices vs. Quantities," Review of Economic Studies 44, 177-182.

[14] Li, Zhe and Jianfei Sun, 2009. "Environmental Policies with Heterogeneous Plants," Manuscript, Shanghai University of Finance and Economics.

[15] Mandell, Svante, 2008. "Optimal Mix of Emissions Taxes and Cap-and-Trade," Journal of Environmental Economics and Management 56, 131-140.

[16] Melitz, Marc J., 2003. "The Impact of Trade on Intra-Industry Reallocations and Aggregate Industry Productivity," Econometrica 71, 1695-1725.

[17] Monnet, C. and T. Temzelides, 2011, "Monetary Emissions Trading Mechanisms," manuscript, Rice University.

[18] Montero, Juan-Pablo, 2005. "Pollution Markets with Imperfectly Observed Emissions," RAND Journal of Economics 36, 645-660.

[19] Pizer, William, 2002. "Combining Price and Quantity Controls to Mitigate Global Climate Change," Journal of Public Economics 85, 409-434.

[20] Requate, Till, 2005. "Environmental Policy under Imperfect Competition - A Survey," working paper 2005-12, University of Kiel.

[21] Shaffer, Sherrill, 1995. "Optimal Linear Taxation of Polluting Oligopolists," Journal of Regulatory Economics 7, 85-100.

[22] Simpson, R. David, 1995. "Optimal Pollution Taxation in a Cournot Duopoly," Environmental and Resource Economics 6, 359-369.

[23] Stern, Nicholas, 2006. "Stern Review on the Economics of Climate Change," UK Treasury.

[24] Weitzman, Martin, 1974. "Prices vs Quantities," Review of Economic Studies 41, 477-491.

[25] Yohe, Gary W., 1978. "Towards a General Comparison of Price Controls and Quantity Controls under Uncertainty," Review of Economic Studies 45, 229-238.

[26] Young, Leslie and James E. Anderson, 1980. "The Optimal Policies for Restricting Trade under Uncertainty," Review of Economic Studies 47, 927-932. 